\title{
Nonadiabatic holonomic quantum computation based on nitrogen-vacancy centers
}

\author{
GuoFu Xu* \\ Department of Physics, Shandong University, Jinan 250100, China
}

Received October 18, 2017; accepted October 19, 2017; published online November 8, 2017

Citation: G. F. Xu, Nonadiabatic holonomic quantum computation based on nitrogen-vacancy centers, Sci. China-Phys. Mech. Astron. 61, 010331 (2018), https://doi.org/10.1007/s11433-017-9123-2

Because of quantum superposition, quantum computation can solve many problems, such as factoring large integers [1] and searching unsorted databases [2,3], much faster than classical computation. To realize practical quantum computation and then gain the desired advantages, a universal set of quantum gates with sufficiently high fidelities are needed. However, various inevitable errors reduce the gate fidelities and finally collapse the computation results, which makes the realizations of quantum computation very challenging. To relax the challenges, different kinds of robust quantum gates have been proposed and holonomic gates are one promising kind. Holonomic gates depend only on evolution paths of a quantum system but not on evolution details. Thus, they are considered to be robust against certain errors and have attracted much attention until now.

Early holonomic gates were realized by evolving the systems adiabatically, which makes the systems expose to the environments for a long time and therefore induces serious decoherence problem. To overcome the long runtime problem, nonadiabatic holonomic quantum computation was recently proposed by realizing the nonadiabatic holonomies in the unitary evolutions $[4,5]$. The realizations of nonadiabatic holonomies can be explained as follows. Consider an $N$-dimensional quantum system exposed to the Hamiltonian $H(t)$ and suppose there is an $L$ dimensional computational subspace $\mathcal{S}(0)$ spanned by the

*Corresponding author (email: sduxgf@163.com) basis vectors $\left\{\left|\phi_{k}(0)\right\rangle\right\}_{k=1}^{L}$. If the initial state is in the subspace $\mathcal{S}(0)$ and the following two conditions are satisfied: (i) $\sum_{k=1}^{L}\left|\phi_{k}(\tau)\right\rangle\left\langle\phi_{k}(\tau)\left|=\sum_{k=1}^{L}\right| \phi_{k}(0)\right\rangle\left\langle\phi_{k}(0)\right|$, and (ii) $\left\langle\phi_{k}(t)|H(t)| \phi_{l}(t)\right\rangle=0, k, l=1, \ldots, L$, then the evolution operator is a nonadiabatic holonomy acting on the computational subspace $\mathcal{S}(0)$, where $\tau$ is the whole evolution time and $\left|\phi_{k}(t)\right\rangle=\boldsymbol{T} \exp \left[-\mathrm{i} \int_{0}^{t} H\left(t^{\prime}\right) \mathrm{d} t^{\prime}\right]\left|\phi_{k}(0)\right\rangle$, with $\boldsymbol{T}$ being time ordering.

From the holonomy conditions (i) and (ii) in the above paragraph, one can see that the first condition guarantees that the action on the computational subspace is unitary while the second condition guarantees that the dynamical phase vanishes and the evolution becomes purely geometric. Generally, making sure the two holonomy conditions are simultaneously satisfied needs considerable effort and is very challenging. Despite this, due to both the robust feature and the high-speed implementation, impressive progress has been made in the field of nonadiabatic holonomic quantum computation. So far, many schemes of realizing nonadiabatic holonomic gates have been put forward for various physical systems. Particularly, nonadiabatic holonomic gates have been experimentally demonstrated with circuit quantum electrodynamics, nuclear magnetic resonance, and nitrogen-vacancy centers in diamond [6-11].

Due to the long electronic spin lifetime, fast initializations, optical readouts, and coherent manipulations even at room temperature, nitrogen-vacancy centers are considered as a promising candidate for quantum information process- 
ing. Until now, many nonadiabatic holonomic quantum computation schemes have been built based on nitrogen-vacancy centers. However, these previous schemes usually employed microwave controls and therefore individual system addressing without crosstalk was difficult. Besides, the quantum gate realizations with microwave controls are not compatible with the initializations and the readouts that are usually achieved in optical ways. To solve these problems, recently Zhou et al. [12] proposed a nonadiabatic holonomic quantum computation scheme based on nitrogen-vacancy centers via all-optical manipulations. In the scheme, the initializations, nonadiabatic holonomic gates, and readouts were all realized by using coherent population trapping and stimulated Raman techniques. The one-qubit gates of the scheme were realized by varying the detunings, amplitudes and phase differences of the applied laser fields, i.e., the single-shot implementations. The two-qubit gates of the scheme were realized by using two ways. One way was confining the needed nitrogen-vacancy centers into one cavity and the other one used muti-cavity couplings.

1 P. W. Shor, SIAM J. Comput. 26, 1484 (1997).

2 L. K. Grover, Phys. Rev. Lett. 79, 325 (1997).

3 G. L. Long, Phys. Rev. A 64, 022307 (2001).

4 E. Sjöqvist, D. M. Tong, L. Mauritz Andersson, B. Hessmo, M. Johansson, and K. Singh, New J. Phys. 14, 103035 (2012).

5 G. F. Xu, J. Zhang, D. M. Tong, E. Sjöqvist, and L. C. Kwek, Phys. Rev. Lett. 109, 170501 (2012).

6 G. Feng, G. Xu, and G. Long, Phys. Rev. Lett. 110, 190501 (2013).

7 A. A. Abdumalikov Jr, J. M. Fink, K. Juliusson, M. Pechal, S. Berger, A. Wallraff, and S. Filipp, Nature 496, 482 (2013).

8 S. Arroyo-Camejo, A. Lazariev, S. W. Hell, and G. Balasubramanian, Nat. Commun. 5, 4870 (2014).

9 C. Zu, W. B. Wang, L. He, W. G. Zhang, C. Y. Dai, F. Wang, and L. M. Duan, Nature 514, 72 (2014).

10 H. Li, Y. Liu, and G. L. Long, Sci. China-Phys. Mech. Astron. 60, 080311 (2017).

11 B. B. Zhou, P. C. Jerger, V. O. Shkolnikov, F. J. Heremans, G. Burkard, and D. D. Awschalom, Phys. Rev. Lett. 119, 140503 (2017).

12 J. Zhou, B. J. Liu, Z. P. Hong, and Z. Y. Xue, Sci. China-Phys. Mech. Astron., 61, 010312 (2018). 